\title{
An Experimental Test Bed for Developing High-Rate Structural Health Monitoring Methods
}

\author{
Bryan Joyce $\mathbb{D}^{1},{ }^{1}$ Jacob Dodson, ${ }^{2}$ Simon Laflamme, ${ }^{3}$ and Jonathan Hong ${ }^{4}$ \\ ${ }^{1}$ Energy Technologies and Materials Division, University of Dayton Research Institute, Eglin AFB, FL 32542, USA \\ ${ }^{2}$ Air Force Research Laboratory (AFRL/RWMF), Eglin AFB, FL 32542, USA \\ ${ }^{3}$ Department of Civil, Construction, and Environmental Engineering, Iowa State University, Ames, IA 50011, USA \\ ${ }^{4}$ Applied Research Associates, Emerald Coast Division, Niceville, FL 32578, USA
}

Correspondence should be addressed to Bryan Joyce; bryan.joyce@udri.udayton.edu

Received 28 February 2018; Revised 20 April 2018; Accepted 2 May 2018; Published 3 June 2018

Academic Editor: Daniele Baraldi

Copyright (c) 2018 Bryan Joyce et al. This is an open access article distributed under the Creative Commons Attribution License, which permits unrestricted use, distribution, and reproduction in any medium, provided the original work is properly cited.

\begin{abstract}
Complex, high-rate dynamic structures, such as hypersonic air vehicles, space structures, and weapon systems, require structural health monitoring (SHM) methods that can detect and characterize damage or a change in the system's configuration on the order of microseconds. While high-rate SHM methods are an area of current research, there are no benchmark experiments for validating these algorithms. This paper outlines the design of an experimental test bed with user-selectable parameters that can change rapidly during the system's response to external forces. The test bed consists of a cantilever beam with electronically detachable added masses and roller constrains that move along the beam. Both controllable system changes can simulate system damage. Experimental results from the test bed are shown in both fixed and changing configurations. A sliding mode observer with a recursive least squares parameter estimator is demonstrated that can track the system's states and changes in its first natural frequency.
\end{abstract}

\section{Introduction}

Researchers have studied a variety of techniques and applications for structural health monitoring (SHM) [1-4]. Much of this interest has focused on civil structures with low frequency dynamics and use SHM techniques that collect and process data on the order of several seconds or longer. Many of these classic methods are too slow to accommodate the need for real-time SHM in the growing number of advanced structures in high-rate, dynamically harsh environments, such as hypervelocity air vehicles, space structures, highspeed turbomachinery, and weapon systems. These structures can experience high-speed impacts $(>4 \mathrm{~km} / \mathrm{s})$ that result in damage propagating through the structures in microseconds $[5,6]$. These high-rate dynamic systems present a number of challenges to contemporary SHM and damage prognosis algorithms including the need for rapid damage detection, robustness to sensor noise, uncertainties in external forces, unknown changes in system parameters, and unmodeled dynamics $[7,8]$.

A number of authors have begun to study the problem of damage detection for high-rate, time-varying systems. Dodson et al. studied SHM at the microsecond timescales using strain energy and wave propagation methods [9]. Kettle, Anton, and collaborators have studied extending electromechanical impedance techniques to the megahertz frequency range for use in real-time damage detection [10-12]. Hong et al. utilized a data-driven, variable input space observer for damage detection $[8,13]$. Dodson et al. previously studied recursive least squares and extended Kalman filter methods for estimating model parameters in simulations of time-varying systems [14, 15]. These works show promise for developing techniques for damage detection in high-rate systems, but sufficient data pertaining to these rapidly changing systems is limited. Such data is needed for developing high-rate SHM techniques and gaining 
insight into detecting structural damage. While researchers have developed benchmark experiments for assessing SHM algorithms in civil structures [16], few have developed test beds with fundamental natural frequencies above tens of hertz and fast changing parameters that can change during the system response.

To address the absence of such data, Abramczyk et al. began developing a test bed with time-varying, usercontrollable parameters for developing real-time SHM algorithms [17]. A summary of this test bed was previously presented by Joyce et al. [18]. Their setup, known as the DROPBEAR (Dynamic Reproduction of Projectiles in Ballistic Environments for Advanced Research), is a cantilever beam featuring two time-varying, user-controllable parameters: the attachment of an electromagnet and the position of roller constrains. Powering off the magnet detaches the mass and simulates a sudden damage or detachment of a system component. A linear actuator moves the rollers and varies the location of the external constraint. Varying either parameter can simulate damage by producing a repeatable, controllable change in the system dynamics. Similar simulated damage strategies can be found elsewhere in the literature, such as attaching masses $[19,20]$, loosing bolts [21, 22], or removing structural components $[16,23]$. Rather than changing configurations between tests, the DROPBEAR's simulated damage can be induced during the system's response as would occur for real damage in high-rate dynamic systems. For repeatability, these temporary changes are preferred over inducing a crack or other permanent defect in the beam. Data sets from the DROPBEAR under known parameter changes can be used to assess the accuracies and speeds of different damage detection or parameter estimation schemes. This allows the DROPBEAR to be an experimental test bed for developing and evaluating methods of real-time damage detection and prognosis.

This paper focuses on modeling the experimental test bed started by Abramczyk et al. $[17,18]$ in different parameter configurations and developing a sliding mode observer (SMO) to demonstrate a model-based state estimator for tracking system changes. SMOs have been successfully implemented for disturbance rejection, disturbance estimation, and structural health monitoring $[24,25]$. SMOs offer robustness to uncertain and unmodeled dynamics which make them attractive for implementation in more complex and difficult to model systems. The results from the SMO are combined with a recursive least squares (RLS) algorithm to estimate the beam's time-varying first natural frequency during the simulated damage. This natural frequency estimation serves as an indicator and measure of the simulated system damage.

The next section provides details of the DROPBEAR setup. An initial analytical model of the system in fixed configurations is derived, discretized using a finite element method, and reduced to a lower order system model for implementation. Next, a SMO is examined to estimate timevarying parameters in the initial model and estimate damage. Modal hammer data from the system with different tip masses and roller positions are compared against predictions from the finite element model. Finally, the SMO is applied to data captured either while the tip mass detaches or while the rollers move along the beam to demonstrate the ability to detect and track simulated damage to this system.

\section{Experimental Setup}

Figure 1 shows the layout of the DROPBEAR experiment. The test bed features a large, rectangular aluminum plate fastened to a tabletop to secure a clamp housing and a clamped steel beam. The steel beam is $51 \mathrm{~mm}$ ( 2 in) wide with a free length of $503 \mathrm{~mm}$ (19.82 in) and a thickness of $6.3 \mathrm{~mm}$ (0.25 in). A PCB 353B17 accelerometer was attached to the beam near the tip. The mass of the beam involved in bending is $1.29 \mathrm{~kg}$. A PCB 086C01 modal hammer was used to excite the beam at different locations along the beam. The accelerometer and modal hammer were connected to a NI-9234 IEPE analog input module seated in a National Instruments (NI) cDAQ-9172 eight-slot chassis. The chassis was connected to a computer with NI LabVIEW to acquire the measured signals, and the collected data was postprocessed in MATLAB to generate frequency response functions (FRFs).

The DROPBEAR has two mechanical parameters that the operator can change during the system response. A detachable electromagnet adds additional mass to any desired location along the beam's length. The electromagnets can be disengaged quickly to simulate a sudden detachment of a system component. Additional mass plates can be bolted to the electromagnet to increase the total added mass. The DROPBEAR also features a sliding cart with rollers on a linear actuator that creates a moveable constraint along the span of the beam. The electromagnet's attachment and the rollers' position can be used as fixed parameters or as variable parameters to simulate damage at any time during testing. The versatility of both components coupled together provides an array of repeatable, fixed, or variable testing configurations. The results in this paper are for the beam in clamped-free (cantilever) boundary conditions, but the setup allows for clamping both ends of the beam to increase the natural frequencies of the system.

\section{Analytical and Numerical Models}

A finite element model (FEM) is developed for the cantilevered beam with different tip masses and a midspan pinned constraint. Because of the large number of elements used in this initial model, a model reduction scheme is adopted to approximate the system's dynamics with a single mode. While this reduced order model will not capture the full transient response of the beam, this model is computationally more efficient to implement in the SMO and captures the necessary dynamics for damage detection as demonstrated later.

3.1. Initial Analytical and Finite Element Models. The transverse displacement of a point along the beam, $w(x, t)$, at location $x$ from the base of the beam and at time $t$ is described by the Euler-Bernoulli equation $[26,27]$

$$
\rho A(x) \frac{\partial^{2} w(x, t)}{\partial t^{2}}+E I \frac{\partial^{4} w(x, t)}{\partial x^{4}}=b(x) u(t),
$$




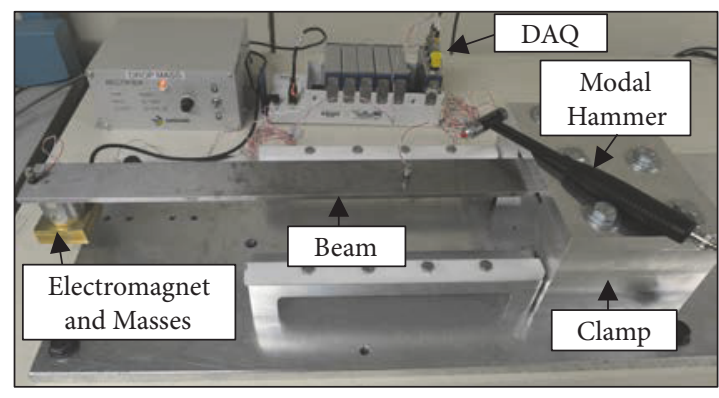

(a)

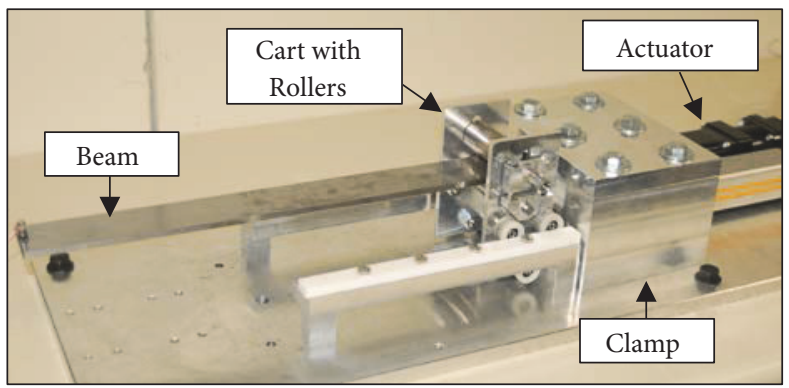

(c)

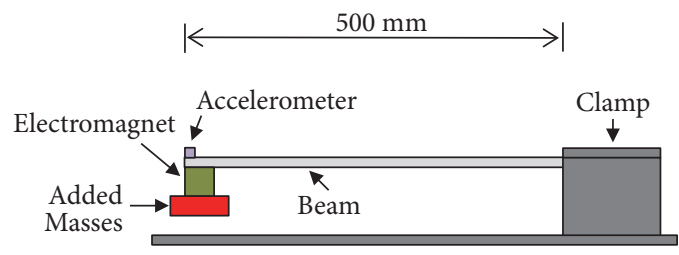

(b)

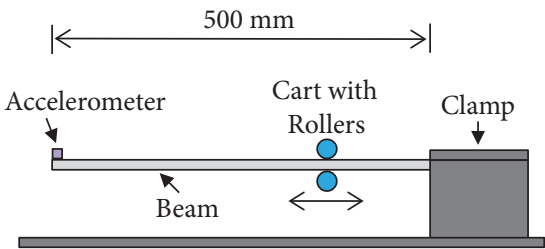

(d)

FIgURE 1: Configurations for the DROPBEAR test bed. Subfigures (a) and (b) show a photo and a schematic of the cantilever beam with detaching electromagnet. Subfigures (c) and (d) show the same setup including the cart with rollers to create a moving pin condition along the span of the beam.

where $\rho A(x)$ is the beam's mass per unit length, $E I$ is the beam's bending stiffness, $u(t)$ is an externally applied force on the beam, and $b(x)$ describes the distribution of the applied force along the length of the beam. The steel beam is assumed to have an elastic modulus of $200 \mathrm{GPa}$ and a density of $7800 \mathrm{~kg} \cdot \mathrm{m}^{-3}$ [28]. The boundary conditions at the clamped end $(x=0)$ and the free end $(x=l)$ are

$$
\begin{gathered}
w(0, t)=0, \\
\frac{\partial w(0, t)}{\partial x}=0, \\
\frac{\partial^{2} w(l, t)}{\partial x^{2}}=0, \\
\frac{\partial^{3} w(l, t)}{\partial x^{3}}=0 .
\end{gathered}
$$

Because of the electromagnet's size, the masses of the electromagnet and added plates at the tip of the beam are included in the spatially varying mass per unit length $\rho A(x)$, i.e.,

$$
\rho A(x)= \begin{cases}\rho_{b} A_{b}, & \text { for } x<l-D_{M} \\ \rho_{b} A_{b}+\frac{M}{D_{M}}, & \text { for } l-D_{M} \leq x \leq l,\end{cases}
$$

where $l$ is the length of the beam, $\rho_{b} A_{b}$ is the mass per unit length of the beam only, $M$ is the total amount of added mass, and $D_{M}$ is the diameter of the electromagnet $(40 \mathrm{~mm})$. The rollers impose a pin condition with an additional torsional stiffness on the beam at a time-varying position $a(t)$. The constrains from the rollers are represented as

$$
\begin{aligned}
w(a(t), t) & =0, \\
\frac{\partial^{2} w(a(t), t)}{\partial x^{2}} & =-k_{\theta} \frac{\partial w(a(t), t)}{\partial x},
\end{aligned}
$$

where $k_{\theta}$ is the rotational stiffness from the rollers.

A FEM of the beam with tip mass and variable roller position was derived from the above equations and boundary conditions using Hermite cubic interpolation polynomials. For a derivation of a FEM of a beam without the rollers, see, for example, Reddy 2005 [29] or Inman 2016 [30]. A MATLAB script is written to compute the mass and stiffness matrices, compute the natural frequencies and mode shapes, and add a damping matrix to account for energy loss in the system. For this application, a total of 400 elements are used. This leads to a model of the form

$$
M \ddot{\mathbf{q}}(t)+S \dot{\mathbf{q}}(t)+K \mathbf{q}(t)=\Gamma_{u} u(t),
$$

where $\mathbf{q}(t)$ is a vector of the transverse displacements and rotations of the finite element nodes, $M$ is the mass matrix, $S$ is a proportional damping matrix, $K$ is the stiffness matrix, and $\boldsymbol{\Gamma}_{u}$ is the influence vector for the force $u(t)$. The overdot denotes time differentiation. The vector $\mathbf{q}(t)$ can be decomposed into modal coordinates as

$$
\mathbf{q}(t)=\Phi \boldsymbol{\eta}(t),
$$


where $\boldsymbol{\eta}(t)$ is the vector of modal coordinates and $\Phi$ is the matrix of mass-normalized mode shapes. From this, the FEM (see (5)) can be written as

$$
\ddot{\boldsymbol{\eta}}(t)+\Lambda_{S} \dot{\boldsymbol{\eta}}(t)+\Lambda \boldsymbol{\eta}(t)=\Phi^{T} \boldsymbol{\Gamma}_{u} u(t),
$$

where the superscript $T$ denotes transposition, $\Lambda=\operatorname{diag}\left(\omega_{r}^{2}\right)$ is a diagonal matrix of the squares of the beam's natural frequencies $\omega_{r}, \Lambda_{S}=\operatorname{diag}\left(2 \zeta_{r} \omega_{r}\right)$ is a diagonal matrix of the modal damping terms, and $\zeta_{r}$ is the modal damping ratio for mode $r$. The modal damping ratios are estimated from the experimental FRFs. The solution in modal coordinates to (7) can be transformed back to physical coordinates using (6).

3.2. Reduced Order Model. For ease of implementation in the SMO discussed in the next section, a model reduction is performed to retain a lower order model. While there are a number of model reduction techniques $[31,32]$, here a modalbased approach is used to approximate the system dynamics with only a few modal coordinates. Let the displacement vector $\mathbf{q}(t)$ be approximated as the response due only to the first mode, i.e.,

$$
\mathbf{q}(t) \approx \phi_{1} \eta_{1}(t)
$$

where $\phi_{1}$ is the first mode shape and $\eta_{1}(t)$ is the modal coordinate for the first mode. From this one mode approximation, only the first modal equation in (7) is considered, i.e.,

$$
\ddot{\eta}_{1}(t)+2 \zeta_{1} \omega_{1} \dot{\eta}_{1}(t)+\omega_{1}^{2} \eta_{1}(t)=\phi_{1}^{T} \boldsymbol{\Gamma}_{u} u(t) .
$$

Next define a state space vector $\mathbf{x}(t)$ as the first modal coordinate and its time derivative, i.e.,

$$
\mathbf{x}(t)=\left[\begin{array}{l}
\eta_{1}(t) \\
\dot{\eta}_{1}(t)
\end{array}\right] .
$$

This leads to the state space equation of the form

$$
\dot{\mathbf{x}}(t)=A \mathbf{x}(t)+\mathbf{B} u(t),
$$

where the state matrix $A$ and the input vector $\mathbf{B}$ are defined as

$$
\begin{aligned}
& A=\left[\begin{array}{cc}
0 & 1 \\
-\omega_{1}^{2} & -2 \zeta_{1} \omega_{1}
\end{array}\right], \\
& \mathbf{B}=\left[\begin{array}{c}
0 \\
\boldsymbol{\phi}_{1}^{T} \boldsymbol{\Gamma}_{u}
\end{array}\right] .
\end{aligned}
$$

For the experiments to follow, the system output $y(t)$ is the measured acceleration at the tip of the beam. This can be written as

$$
y(t)=\frac{\partial^{2} w(l, t)}{\partial t^{2}} .
$$

In terms of the first modal coordinate, the output is approximately

$$
y(t) \approx \Gamma_{y}^{T} \phi_{1} \ddot{\eta}_{1},
$$

where the vector $\Gamma_{y}$ is one at the element corresponding to the translation degree of freedom at the beam tip and zero for all other entries, i.e., $\Gamma_{y}=\left[\begin{array}{lllllll}0 & 0 & 0 & \cdots & 0 & 1 & 0\end{array}\right]^{T}$. In state space representation, the output is written as

$$
y(t)=C \mathbf{x}(t)+D u(t),
$$

where the output matrix $C$ and the direct feedthrough term $D$ are defined as

$$
\begin{aligned}
& C=\Gamma_{y}^{T} \phi_{1}\left[-\Lambda-\Lambda_{S}\right], \\
& D=\Gamma_{y}^{T} \phi_{1} \phi_{1}^{T} \Gamma_{u} .
\end{aligned}
$$

\section{Model-Based State and Parameter Estimation}

An SMO is derived utilizing the reduced order system model. The results from this observer are combined with an RLS algorithm to estimate the natural frequency of the beam during parameter changes.

4.1. State Observer with Uncertain Plant Dynamics. An SMO is derived following work outlined by Shtessel et al. [24]. The reduced order model derived previously (see (11) and (15)) is rewritten to include uncertain dynamics from a parameter change as

$$
\begin{aligned}
\dot{\mathbf{x}}(t) & =A \mathbf{x}(t)+\mathbf{B} u(t)+\mathbf{M} \xi(t, \mathbf{x}(t), u(t)), \\
y & =C \mathbf{x}(t)+D u(t),
\end{aligned}
$$

where the term $\mathbf{M} \xi(t, \mathbf{x}(t), u(t))$ represents the time-varying, uncertain dynamics. Next, introduce the new coordinate $\mathbf{z}(t)=T \mathbf{x}(t)$ with

$$
T=\left[\begin{array}{c}
N_{C}^{T} \\
C
\end{array}\right],
$$

where $N_{C}$ is a matrix whose columns span the null space of $C$. In the new coordinates, the state space equations become

$$
\begin{aligned}
\dot{\mathbf{z}}(t) & =A_{z} \mathbf{z}(t)+\mathbf{B}_{z} u(t)+\mathbf{M}_{z} \xi\left(t, T^{-1} \mathbf{z}(t), u\right), \\
y & =C_{z} \mathbf{z}(t)+D u(t),
\end{aligned}
$$

with the transformed matrices $A_{z}=T A T^{-1}, \mathbf{B}_{z}=T \mathbf{B}, C_{z}=$ $C T^{-1}$, and $\mathbf{M}_{z}=T \mathbf{M}$. These matrices can be partitioned as

$$
\begin{aligned}
\mathbf{z}(t) & =\left[\begin{array}{c}
z_{1}(t) \\
y(t)-D u(t)
\end{array}\right], \\
A & =\left[\begin{array}{ll}
A_{11} & A_{12} \\
A_{21} & A_{22}
\end{array}\right], \\
\mathbf{B}_{z} & =\left[\begin{array}{l}
B_{z 1} \\
B_{z 2}
\end{array}\right], \\
C_{z} & =\left[\begin{array}{ll}
0 & 1
\end{array}\right], \\
\mathbf{M}_{z} & =\left[\begin{array}{l}
M_{z 1} \\
M_{z 2}
\end{array}\right] .
\end{aligned}
$$


The new state vector $\mathbf{z}(t)$ has two components: an unmeasured state $z_{1}(t)$ and a state that is the measured tip acceleration $y(t)$ without the direct feedthrough term $D u(t)$. The $\mathrm{SMO}$ in the transformed coordinates has the structure

$$
\begin{aligned}
\dot{\mathbf{z}}(t) & =A_{z} \widehat{\mathbf{z}}(t)+\mathbf{B}_{z} u(t)+\mathbf{G}_{z} v(t), \\
\hat{y}(t) & =C_{z} \widehat{\mathbf{z}}(t)+D u(t),
\end{aligned}
$$

where $\widehat{y}(t)$ is the estimated measurement, $\widehat{\mathbf{z}}(t)$ is the estimated state vector, $\mathbf{G}_{z}$ is a gain vector to be determined, and the discontinuous injection term $v(t)$ is defined as

$$
v(t)=\rho \operatorname{sgn}(y-\widehat{y}),
$$

where $\rho$ is a positive scalar to be determined and sgn is the signum function. The gain $\mathbf{G}_{z}$ has the structure

$$
\mathbf{G}_{z}=\left[\begin{array}{c}
L \\
-1
\end{array}\right] \text {. }
$$

As shown in Shtessel et al. [24], the estimated states of this observer will converge to the true states if the gain $L$ is chosen to stabilize the matrix $A_{11}+L A_{21}$ and $\rho$ is selected large enough such that

$$
\rho>\left|A_{21} \bar{e}_{1}+A_{22} \bar{e}_{y}+M_{z 2} \bar{\xi}\right|+\mu,
$$

where $\bar{e}_{1}$ is a bound on the estimation error of the unmeasured state $z_{1}, \bar{e}_{y}$ is a bound on the output error, $\bar{\xi}$ is a bound on the model uncertainty, and $\mu$ is a positive scalar. Finally, for application to the DROPBEAR system, the observer in (21) is implemented in the state space coordinates $\mathbf{x}(t)$ as

$$
\begin{aligned}
\dot{\hat{\mathbf{x}}}(t) & =A \widehat{\mathbf{x}}(t)+\mathbf{B} u(t)+\mathbf{G} v(t), \\
\hat{y}(t) & =C \widehat{\mathbf{x}}(t)+D u(t),
\end{aligned}
$$

where $\widehat{\mathbf{x}}(t)=T^{-1} \widehat{\mathbf{z}}$ and $\mathbf{G}=T^{-1} \mathbf{G}_{z}$.

4.2. Natural Frequency Estimation from Least Squares Regression. The observer feedback term $\mathbf{G} v(t)$ in the SMO can be used to estimate damage or time-varying parameters. Let the time-varying first natural frequency of the beam $\omega_{1}(t)$ be written as

$$
\left(\omega_{1}(t)\right)^{2}=\omega_{0}^{2}+\theta(t),
$$

where $\omega_{0}$ is the assumed first natural frequency in the model and $\theta(t)$ is a time-varying parameter indicating the change in natural frequency. $\theta(t)$ may be nonzero due to model error and may change in time when damage occurs that alters the first natural frequency. If there is a change in the natural frequency and assuming that there is little change in damping or force influence terms, then the system model in (11) can be written as

$$
\dot{\mathbf{x}}(t)=A_{0} \mathbf{x}(t)+\mathbf{B} u(t)+\left[\begin{array}{c}
0 \\
-1
\end{array}\right] \eta_{1}(t) \theta(t),
$$

where $A_{0}$ is the original state matrix in the model given by

$$
A_{0}=\left[\begin{array}{cc}
0 & 1 \\
-\omega_{0}^{2} & -2 \zeta_{1} \omega_{0}
\end{array}\right] .
$$

By comparing this to the uncertain system equations in (17), after the estimated states converge to the true states, one can equate the perturbation term in (17) to the equivalent form of the feedback term in (27), i.e.,

$$
\mathbf{G} F[v(t)] \approx\left[\begin{array}{c}
0 \\
-1
\end{array}\right] F\left[\eta_{1}(t)\right] \theta(t),
$$

where $F$ denotes a low-pass filter applied to the signals to smooth the discontinuities from the injection signal $v(t)$. This leads to an RLS algorithm to estimate a value of $\theta(t)$ that best minimizes the error to (29) [33]. The estimated parameter $\hat{\theta}(k)$ at discrete time $t(k)$ is written as

$$
\widehat{\theta}(k)=\min _{\theta}\left(\sum_{i=1}^{k} \lambda^{k-i}\left|G_{2} F[v(i)]+F\left[\widehat{\eta}_{1}(i)\right] \theta\right|^{2}\right),
$$

where $G_{2}$ is the second component of $\mathbf{G}$ and $\lambda$ is a forgetting factor between zero and one that allows the parameter estimate to adapt to system changes. A smaller value of $\lambda$ results in faster convergence of the parameter estimate but reduces robustness to measurement noise.

\section{Modal Testing in Fixed Configurations}

First the DROPBEAR setup was tested in various fixed parameter conditions to validate the finite element model. Frequency response functions (FRFs) are computed from a set of impact hammer tests. These FRFs and the beam's natural frequencies are compared against predictions from the model. This validated model was then implemented in the SMO to track the system under time-varying parameters.

5.1. Modal Testing in Fixed Mass Configurations. The beam was first tested with various masses attached near the tip of the beam and the cart and rollers removed. Two added mass configurations were tested. The first consisted of the electromagnet alone (mass of $0.259 \mathrm{~kg}$ ), and the second consisted of the electromagnet, three mass plates, and the adjoining bolt (total mass of $0.695 \mathrm{~kg}$ ). The beam was struck by the modal hammer at $483 \mathrm{~mm}$ (19 in) from the clamp. The beam was struck five times per test with enough time between each strike to observe the full decay in the response. The measured, time-domain data was processed in MATLAB to compute the experimental FRFs using the $H_{1}$ estimation method $[34,35]$. For each mass configuration, the natural frequencies predicted by the FEM were validated against natural frequencies calculated using the FRFs derived from experimental data. Figure 2 plots the FRFs from experimental data alongside those determined by the finite element model. The figure also shows the coherence, which is a measure of the linear dependency of the output tip acceleration due to the input force from the modal hammer as a function of frequency [35]. The natural frequencies for the three different 
TABLE 1: Finite element and experimental natural frequencies for the DROPBEAR with no added mass $(M=0 \mathrm{~kg})$, adding the electromagnet near the beam tip $(M=0.259 \mathrm{~kg})$, and with the electromagnet and added mass plates $(M=0.695 \mathrm{~kg})$. Percent errors are relative to the experimentally obtained natural frequencies.

\begin{tabular}{lcccc}
\hline \multirow{2}{*}{ Added Mass $(M)$} & Mode & $\begin{array}{c}\text { Experimental } \\
\text { Natural Frequency [Hz] }\end{array}$ & \multicolumn{2}{c}{$\begin{array}{c}\text { Finite Element } \\
\text { Error }\end{array}$} \\
\hline \multirow{2}{*}{$0 \mathrm{~kg}$} & 1 & 19.6 & $0.8 \%$ \\
& 2 & 124.5 & 19.8 & $-0.5 \%$ \\
\hline \multirow{2}{*}{$0.259 \mathrm{~kg}$} & 3 & 350.0 & 346.8 & $-0.9 \%$ \\
& 1 & 15.3 & 15.2 & $-0.7 \%$ \\
& 2 & 106.9 & 106.6 & $-0.3 \%$ \\
$0.695 \mathrm{~kg}$ & 3 & 311.8 & 307.2 & $-1.5 \%$ \\
& 1 & 11.6 & 11.6 & $0.3 \%$ \\
& 2 & 91.5 & 90.6 & $-1.0 \%$ \\
\hline
\end{tabular}
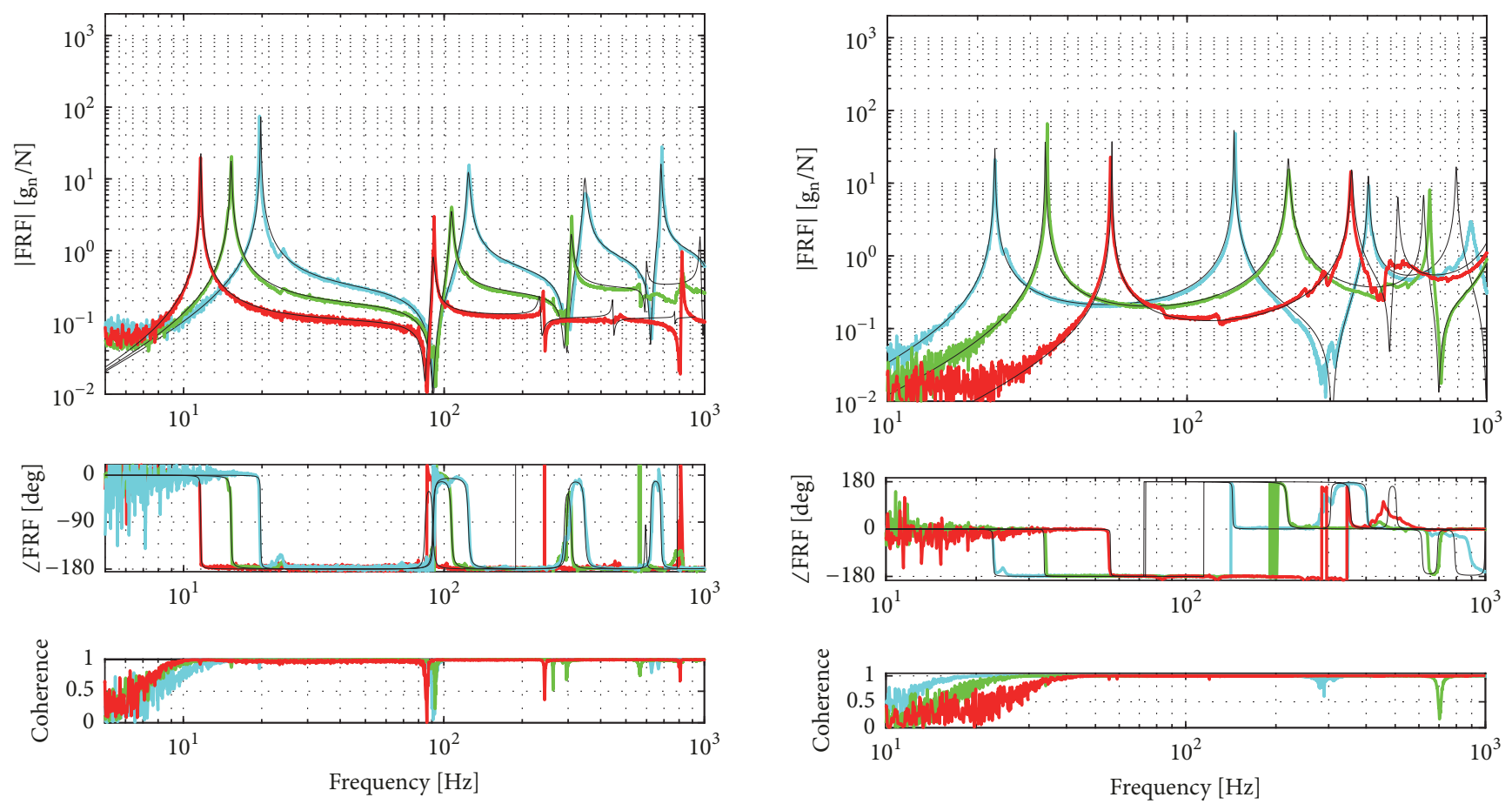

$$
\begin{array}{ll}
\text { Data }(\mathrm{M}=0 \mathrm{~kg}) & \text { Data }(\mathrm{M}=0.695 \mathrm{~kg}) \\
\text { Model }(\mathrm{M}=0 \mathrm{~kg}) & \text { Model }(\mathrm{M}=0.695 \mathrm{~kg}) \\
\text { Data }(\mathrm{M}=0.259 \mathrm{~kg}) & \\
\text { Model }(\mathrm{M}=0.259 \mathrm{~kg}) &
\end{array}
$$

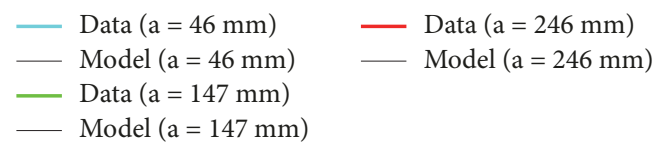

FIGURE 2: Frequency response functions (FRFs) for the bare beam ( $M=0 \mathrm{~kg})$, beam with the electromagnet $(M=0.259 \mathrm{~kg})$, and beam with the electromagnet and added mass plates $(M=0.695 \mathrm{~kg})$. Data is from the tip accelerometer and modal hammer impacts at $483 \mathrm{~mm}$ (19 in) from the base. Note that $g_{n}=9.81 \mathrm{~m} \cdot \mathrm{s}^{-2}$.

tip masses are listed in Table 1. The FRFs from the model and the data show good agreement. Adding more mass to the beam decreased the natural frequencies as expected. It should be noted that model updating could be used to refine the initial model parameters and improve the model's accuracy. The small errors between the experimental and finite element natural frequencies indicate that the model captures the

FIGURE 3: Frequency response functions (FRFs) for the DROPBEAR with the rollers in three different positions: $46 \mathrm{~mm}, 147 \mathrm{~mm}$, and $246 \mathrm{~mm}$. Data is from the tip accelerometer and impacts at $305 \mathrm{~mm}$ (12 in) from the base. Note that $g_{n}=9.81 \mathrm{~m} \cdot \mathrm{s}^{-2}$.

behavior of the baseline system with fixed parameters and could predict the beam's response once the mass detaches.

5.2. Modal Testing in Fixed Roller Positions. Next, the DROPBEAR system was tested without the added masses and with the rollers in different positions. Modal hammer tests were repeated for the rollers in three positions spanning the full displacement range of the actuator. Figure 3 plots the FRFs 
TABLE 2: Finite element and experimental natural frequencies for the DROPBEAR with the rollers at distances from the clamped end (a) of $46 \mathrm{~mm}, 147 \mathrm{~mm}$, and $246 \mathrm{~mm}$. Percent errors are relative to the experimentally obtained natural frequencies.

\begin{tabular}{lcccr}
\hline Cart Position $(a)$ & Mode & $\begin{array}{c}\text { Experimental } \\
\text { Natural Frequency }[\mathrm{Hz}]\end{array}$ & Finite Element & Natural Frequency $[\mathrm{Hz}]$ \\
\hline \multirow{2}{*}{$46 \mathrm{~mm}$} & 1 & 22.9 & 22.8 & $-0.4 \%$ \\
& 2 & 144.8 & 143.4 & $-1.0 \%$ \\
\hline \multirow{3}{*}{$147 \mathrm{~mm}$} & 3 & 404.0 & 402.7 & $-0.3 \%$ \\
& 1 & 33.2 & 33.7 & $1.5 \%$ \\
& 2 & 218.6 & 217.7 & $-0.4 \%$ \\
$246 \mathrm{~mm}$ & 3 & 645.2 & 615.6 & $-4.6 \%$ \\
& 1 & 55.6 & 56.6 & $1.8 \%$ \\
& 2 & 352.1 & 355.2 & $0.9 \%$ \\
\hline
\end{tabular}

computed from experimental data and from the FEM. The model treated the rollers as a midspan pinned condition with an additional torsional stiffness $\left(k_{\theta}\right)$ of $900 \mathrm{~N} \mathrm{~m} \mathrm{rad}^{-1}$. This stiffness was manually estimated from the experimental FRF near the first natural frequency with the rollers in the $46 \mathrm{~mm}$ position. Table 2 lists the natural frequencies from the experimental FRFs and those predicted by the model for the three roller positions. The natural frequencies and FRFs from the model show good agreement with the experimental results. Further model updating could be conducted to improve the model accuracy.

5.3. Dropping Mass Experiments. With the FEM validated for different fixed parameters, the system was examined with time-varying conditions. Two experiments with time-varying parameters were conducted. The first involved detaching the electromagnet during the beam's ringdown from an impact. The second experiment used the linear actuator to move the rollers along the beam to replicate changes in external constraints on the beam and create a continuous change in system parameters. The SMO was used to track the varying first natural frequency of the beam during both parameter changes. For both experiments, the RLS algorithm for estimating $\theta$ used a forgetting factor $\lambda$ of 0.999 and a fourth-order Butterworth filter with a corner frequency of $50 \mathrm{~Hz}$ for the smoothing filter $F$.

Figure 4 plots the data from the tip accelerometer during the mass detachment. The beam was given an initial impulse and allowed to ringdown. Then, the electromagnet was powered off. There was a sudden spike in the tip acceleration when the magnet was released. The beam continued to vibrate for about one cycle at the original $12 \mathrm{~Hz}$ frequency before there was a noticeable change in the oscillation frequency. This transition occurs over approximately $86 \mathrm{~ms}$. While the mass detachment was nearly instantaneous, the structural waves had to propagate from one end of the beam to the other and back before the standing waves or structural modes formed. At higher natural frequencies, this time scale of change would be less than $86 \mathrm{~ms}$.

Figure 5 shows the experimental results and estimations from the SMO during the mass drop. Figure 5(c) plots

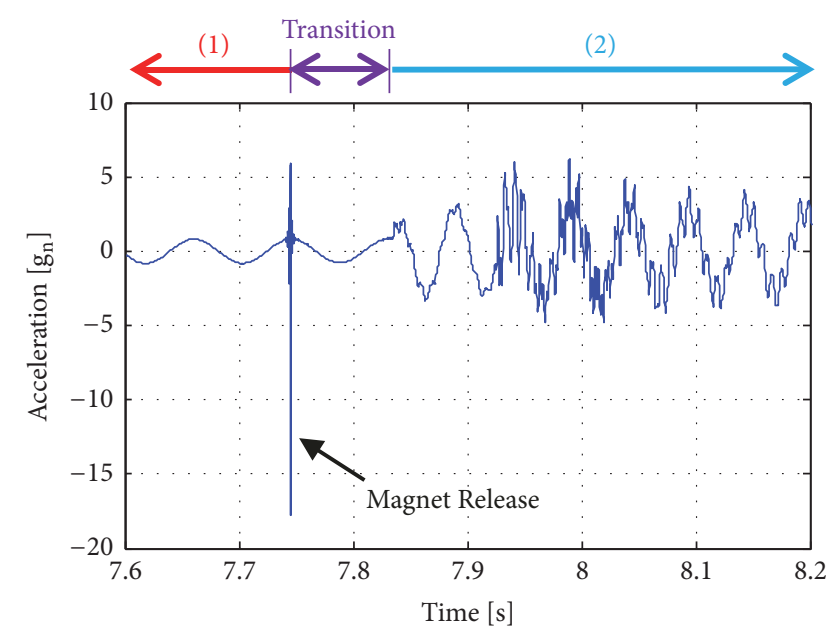

FIGURE 4: Detailed view of tip acceleration after the mass releases from the beam. There is a transition period between the beam in the first configuration (1) and the second configuration (2).

data from the tip accelerometer recorded during the mass drop, and Figure 5(d) plots a power spectrogram of this data. The power spectrogram is based on a short-time Fast Fourier Transform using a moving Hanning window. The spectrogram highlights the time-varying frequency spectrum of the acceleration signal. Here, the sampling rate was 5,120 samples per second, the spectrogram window had a length of $1 \mathrm{~s}$, and the overlap of spectrogram windows was $99 \%$. The spectrogram initially shows a dominating frequency near $12 \mathrm{~Hz}$ corresponding to the first natural frequency of the beam with $0.695 \mathrm{~kg}$ of added mass. The mass removal increased the natural frequencies of the beam and caused a rapid increase in the dominate frequency in the observed spectrogram in Figure 5(c). The remainder of the signal was concentrated around $20 \mathrm{~Hz}$ corresponding to the first natural frequency of the bare beam.

The SMO was applied to the data from the accelerometer at the tip of the beam. The SMO used a beam model with the electromagnet only (no additional mass plates). The estimated output from the SMO is also shown in Figures 5(c) 


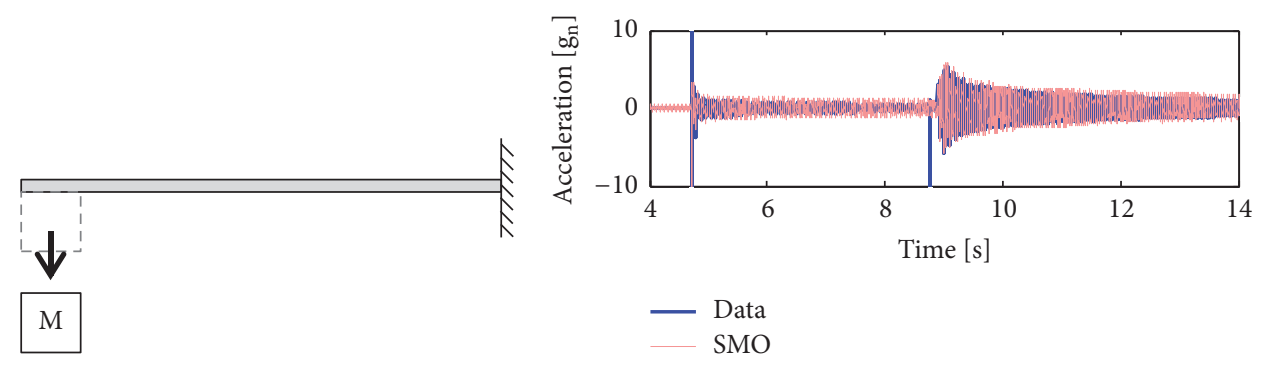

(a)

(b)

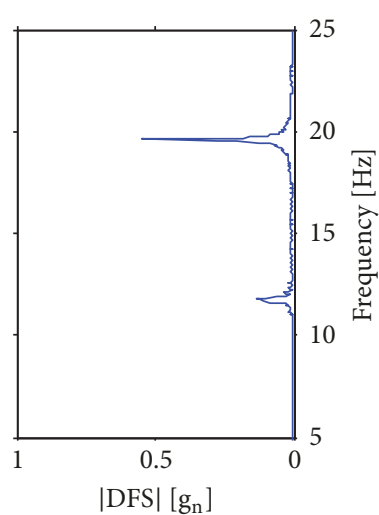

(c)

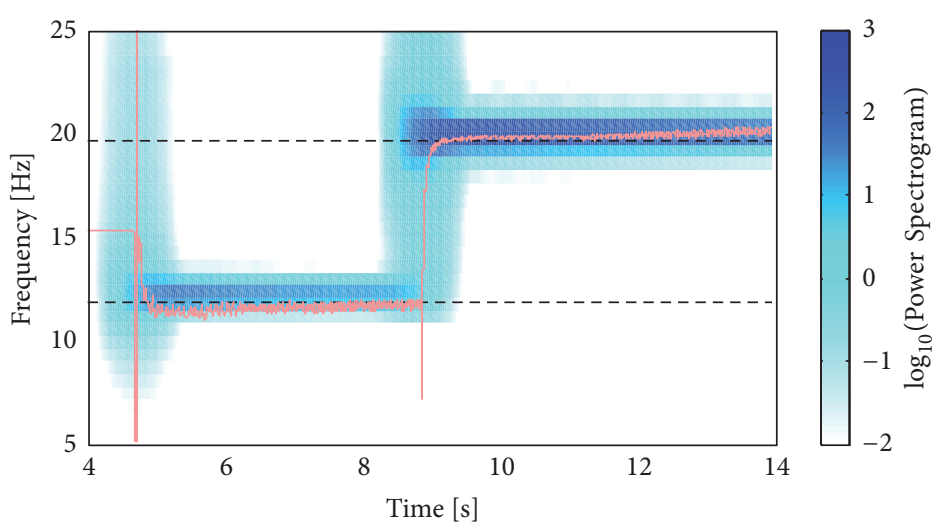

(d)

Figure 5: Results from the mass drop experiment. (a) Diagram of the mass dropping from the beam. (b) Amplitude of the discrete Fourier series (DFS) of the tip acceleration. (c) Time history of the tip acceleration. (d) Spectrogram of the tip acceleration when the mass drops. The horizontal dashed lines indicate the frequencies for the first natural frequency with and without the added mass.

and 5(d). Before the initial impact, the estimator operated on only signal noise and could not accurately update its parameters. Joyce et al. [15] illustrated this issue for parameter estimation in simulations of time-varying mechanical systems. After the initial impulse from the impact hammer, the estimated first natural frequency decreased toward the correct value for a beam with electromagnet and added mass plates. When the electromagnet was detached, the estimated first natural frequency quickly increased toward the correct value of the beam with no mass. Both changes in estimated natural frequency settled near the correct values after about 0.2 seconds.

5.4. Time-Varying Roller Positions. Next, the DROPBEAR is tested with the rollers moving along its length during the beam's ringdown, as illustrated in Figure 6(a). As the rollers move along the beam, they create a moving pinned condition along the span of the beam. The beam was first struck by the impact hammer, then the rollers moved from $46 \mathrm{~mm}$ to $195 \mathrm{~mm}$ over a $1.5 \mathrm{~s}$ duration, the rollers sat at the $195 \mathrm{~mm}$ position for $1 \mathrm{~s}$, and then the rollers returned to the $46 \mathrm{~mm}$ position over $0.5 \mathrm{~s}$. The SMO was again applied to data from the accelerometer to track the states and estimate the first natural frequency.

Figure 6 plots the time response of the tip accelerometer, the power spectrogram of the data, and the results from the SMO. Over this displacement range of the linear actuator, the first natural frequency of the beam increased when the rollers moved away from the base and decreased when they moved back. The SMO was able to track the changing natural frequency over this range of roller motion. The estimated natural frequency from the observer initially converged to the correct value before the rollers began to move. As the rollers moved away from the base (increasing natural frequency), the estimated first natural frequency tracked the actual value with a maximum error of $2.2 \mathrm{~Hz}$ and a normalized-root-meansquare error (NRMSE) of 3.4\%. During the faster return toward the base, the parameter estimation lagged behind the actual value but was able to converge to the correct value in approximately $1 \mathrm{~s}$. During the rollers return (decreasing natural frequency), the maximum error was $10.7 \mathrm{~Hz}$ and the NRMSE was $16.3 \%$.

\section{Conclusions and Future Work}

There is a need for an experimental test bed for developing and demonstrating high-rate damage detection methods. The DROPBEAR serves as a unique test bed capable of producing repeatable, time-varying system conditions that can assist in evaluating real-time SHM and state estimation algorithms for high-rate systems. Results from a finite element model of the DROPBEAR showed good agreement to the experimentally obtained FRFs with different tip masses and cart positions. Detaching the electromagnet mimicked sudden damage to the DROPBEAR and illustrated the speed at which the simulated damage affected the system's response. The 


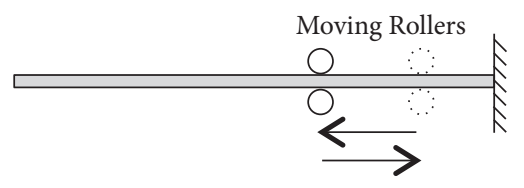

(a)

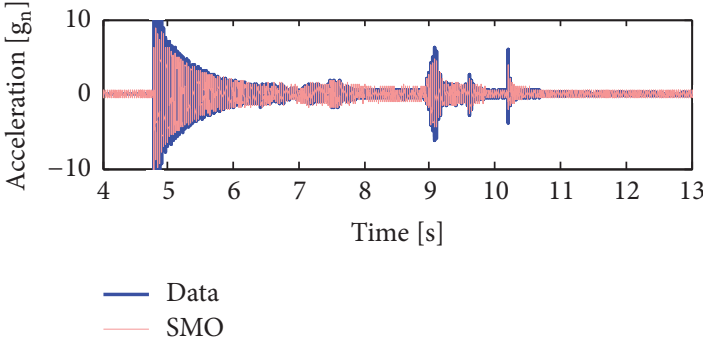

(b)

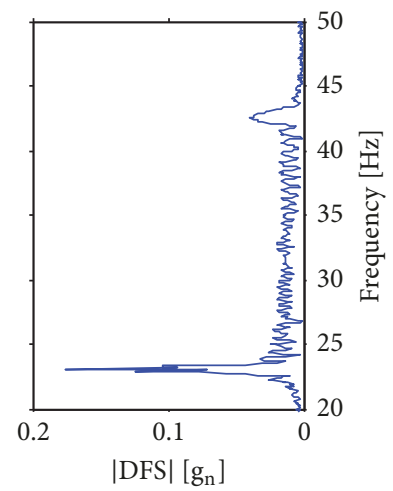

(c)

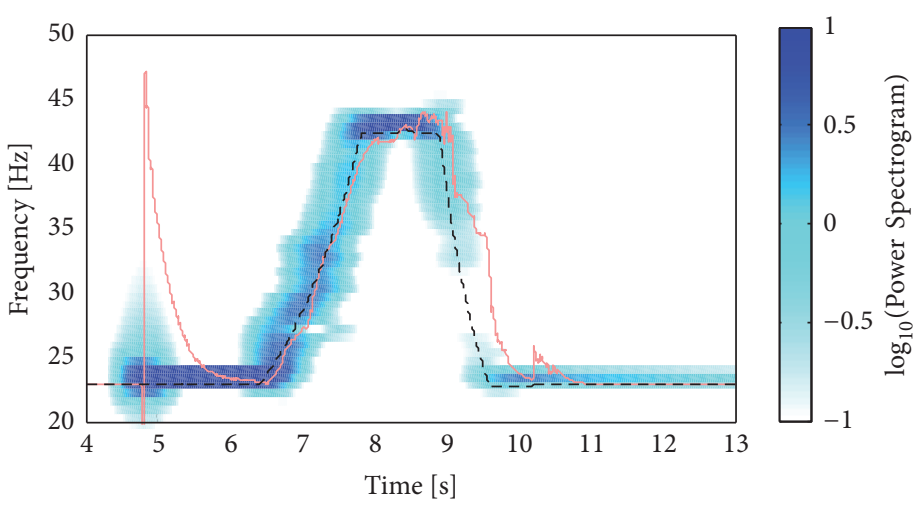

(d)

Figure 6: Results from the moving roller experiment. (a) Diagram of the beam with moving rollers. (b) Amplitude of the discrete Fourier series (DFS) of the tip acceleration. (c) Time history of the tip acceleration. (d) Spectrogram of the tip acceleration while the rollers move. The dashed line shows the natural frequency estimated from the cart position obtained from the encoder on the linear actuator.

moving roller test showed how the natural frequencies of the DROPBEAR can also be adjusted continuously at a user definable range and rate to mimic continuously progressing damage or changes in external constraints.

For both varying parameter tests, a SMO was able to track the time-varying first natural frequency. This measure of natural frequency change could be used to detect and quantify system damage in application. The demonstrated ability of an SMO to quickly detect changes in the beam's first natural frequency shows promise for applying other SHM and damage prognosis techniques to this system. Data from the DROPBEAR will guide developing and evaluating algorithms for real-time state estimation, system identification, and damage detection of more complex, high-rate dynamic systems such as high-speed airframes, space structures, car crashes, or civil structures subjected to impacts.

The current DROPBEAR model is limited to fixed parameters. To fully understand the dynamics involved in the timevarying system, future work will extend the FEM presented here to study a model with time-varying parameters. It should also be noted that the DROPBEAR's parameter changes alter the beam's mode shapes as well as its natural frequencies. This means the modal decomposition and resulting state space matrices will vary as a function of the attached mass or roller position. The SMO can compensate for some of this model uncertainty; however detailed analysis of the DROPBEAR with changing mode shapes $\Phi$, output matrix $C$, and direct feedthrough term $D$ is left for future work.

\section{Data Availability}

Data from this manuscript may be made available upon request to the authors.

\section{Disclosure}

A brief introduction of the DROPBEAR test bed was presented at the International Modal Analysis Conference (IMAC) XXXVI in February 2018 [18]. Any opinions, findings, and conclusions or recommendations expressed in this material are those of the authors and do not necessarily reflect the views of the United States Air Force (Distribution A. Approved for public release; distribution unlimited (96TW2018-0094)).

\section{Conflicts of Interest}

The authors declare that there are no conflicts of interest regarding the publication of this paper.

\section{Acknowledgments}

The material in this paper is based upon work supported by the Air Force Office of Scientific Research under Award no. FA9550-17RWCOR503 and Award no. FA9550-71-1-0131. 


\section{References}

[1] S. W. Doebling, C. R. Farrar, M. B. Prime, and D. W. Shevitz, "Damage identification and health monitoring of structural and mechanical systems from changes in their vibration characteristics: a literature review," The Shock and Vibration Digest, vol. 30, No. LA-13070-MS, 1996.

[2] S. W. Doebling, C. R. Farrar, and M. B. Prime, "A summary review of vibration-based damage identification methods," The Shock and Vibration Digest, vol. 30, no. 2, pp. 91-105, 1998.

[3] E. P. Carden and P. Fanning, "Vibration based condition monitoring: a review," Structural Health and Monitoring, vol. 3, no. 4, pp. 355-377, 2004.

[4] C. Farrar and K. Worden, Structural health monitoring: a machine learning perspective, John Wiley Sons, Ltd, Chichester, West Sussex, UK, 2012.

[5] C. Stein, R. Roybal, P. Tlomak, and W. Wilson, "A Review of Hypervelocity Debris Testing at the Air Force Research Laboratory," International Journal of Space Debris, vol. 2, no. 4, pp. 331-356, 2000.

[6] R. Hallion, C. Bedke, and M. Schanz, "Hypersonic weapons and US national security, a 21st century breakthrough," Tech. Rep., Mitchell Institute for Aerospace Studies, Air Force Association, 2016.

[7] R. Lowe, J. Dodson, and J. Foley, "Microsecond prognostics and health monitoring," IEEE Reliability Society Newsletter, vol. 60, pp. 1-5, 2014.

[8] J. Hong, S. Laflamme, J. Dodson, and B. Joyce, "Introduction to State Estimation of High-Rate System Dynamics," Sensors, vol. 18, no. 2, p. 217, 2018.

[9] J. C. Dodson, D. J. Inman, and J. R. Foley, "Microsecond structural health monitoring in impact loaded structures," Health Monitoring of Structural and Biological Systems, vol. 7295, 2009.

[10] J. Dodson, R. Kettle, and S. R. Anton, "Microsecond state detection and prognosis using high-rate electromechanical impedance," in Proceedings of the IEEE Prognostics and Health Management Conference (PHM'16), Ottawa, ON, Canada, 2016.

[11] R. A. Kettle, A. J. Dick, J. C. Dodson, J. R. Foley, and S. R. Anton, "Real-Time State Detection in Highly Dynamic Systems," in Proceedings of the 34th IMAC, vol. 8, pp. 27-34, Springer.

[12] R. A. Kettle, J. C. Dodson, and S. R. Anton, "High-Frequency Impedance Measurements for Microsecond State Detection," in Dynamics of Civil Structures, vol. 2, pp. 235-242, Springer International Publishing, 2017.

[13] J. Hong, S. Laflamme, L. Cao, and J. Dodson, "Variable input observer for structural health monitoring of high-rate systems," in Proceedings of the AIP Conference, vol. 1806, 2017.

[14] J. Dodson, B. Joyce, J. Hong, S. Laflamme, and J. Wolfson, "Microsecond State Monitoring of Nonlinear Time-Varying Dynamic Systems," in Proceedings of the ASME 2017 Conference on Smart Materials, Adaptive Structures and Intelligent Systems, pp. 18-20, Utah, USA.

[15] B. Joyce, J. Hong, J. Dodson, J. Wolfson, and S. Laflamme, "Adaptive observers for structural health monitoring of highrate, time-varying dynamic systems," in International Modal Analysis Conference (IMAC) XXXVI, pp. 12-15, Orlando, FLA, USA, 2018.

[16] E. A. Johnson, H. F. Lam, L. S. Katafygiotis, and J. L. Beck, "Phase I IASC-ASCE structural health monitoring benchmark problem using simulated data," Journal of Engineering Mechanics, vol. 130, no. 1, pp. 3-15, 2004.
[17] S. Abramczyk, H. Karsten, J. Markl, R. Minger, and E. Passmore, "Capstone design final report - DROPBEAR appartus," Tech. Rep., Michigan Technological University, 2017.

[18] B. Joyce, K. Greenoe, J. Dodson et al., "An experimental test bed with time-varying parameters for developing highrate structural health monitoring methods," in Proceedings of the International Modal Analysis Conference (IMAC) XXXVI, Orlando, FL, 2018, http://sem.org/Files/events/XXXVI_AdvanceProgram_web.pdf.

[19] C. Pitchford, T. Kundu, B. L. Grisso, and D. J. Inman, Impedancebased structural health monitoring of wind turbine blades [Ph.D. thesis], Virginia Tech, 2007.

[20] P. J. Fanning and E. P. Carden, "Experimentally Validated Added Mass Identification Algorithm Based on Frequency Response Functions," Journal of Engineering Mechanics, vol. 130, no. 9, pp. 1045-1051, 2004.

[21] J. Li, Structural health monitoring of an in-service highway bridge with uncertainties [Ph.D. thesis], University of Connecticut, 2014.

[22] G. Park, H. H. Cudney, and D. J. Inman, "Impedance-based health monitoring of civil structural components," Journal of Infrastructure Systems, vol. 6, no. 4, pp. 153-160, 2000.

[23] G. Manson, K. Worden, and D. Allman, "Experimental validation of a structural health monitoring methodology: Part III. Damage location on an aircraft wing," Journal of Sound and Vibration, vol. 259, no. 2, pp. 365-385, 2003.

[24] Y. Shtessel, C. Edwards, L. Fridman, and A. Levant, Sliding Mode Control and Observation, Birkhäuser, Springer, New York, NY, USA, 2014.

[25] M. Kim, "Simultaneous Structural Health Monitoring and Vibration Control of Adaptive Structures Using Smart Materials," Shock and Vibration, vol. 9, no. 6, pp. 329-339, 2002.

[26] D. Inman, Engineering vibration, Prentice Hall, NJ, USA, 4th edition, 2014.

[27] A. Chopra, "Dynamics of structures: theory and applications to earthquake engineering," in Prentice-hall International Series I Civil Engineering and Engineering Mechanics, Pearson PrenticeHall, Prentice-Hall, Englewood Cliffs, NJ, USA, 2017.

[28] MatWeb, "Steels, general properties," http://www.matweb.com/ search/datasheet.aspx.

[29] J. Reddy, "An introduction to the finite element method," in McGraw-Hill Series in Mechanical Engineering, McGraw-Hill Education, New York, NY, USA, 3rd edition, 2005.

[30] D. Inman, Vibration with control, John Wiley Sons, Ltd, Chichester, UK, 2006.

[31] D. Bonvin and D. A. Mellichamp, "A unified derivation and critical review of modal approaches to model reduction," International Journal of Control, vol. 35, no. 5, pp. 829-848, 1982.

[32] A. C. Antoulas, D. C. Sorensen, and S. Gugercin, "A survey of model reduction methods for large-scale systems," in Structured matrices in mathematics, computer science, and engineering, I (Boulder, CO, 1999), vol. 280 of Contemp. Math., pp. 193-219, Amer. Math. Soc., Providence, RI, 2001.

[33] K. Åström and B. Witternmark, Adaptive control, AddisonWesley Longman Publishing Co., Inc, Boston, Mass, USA, 1994.

[34] R. J. Allemang and D. L. Brown, "Experimental Modal Analysis and Dynamic Component Synthesis. Volume 1. Summary of Technical Work," Defense Technical Information Center, 1987.

[35] D. J. Ewins, Modal testing: theory, practice, and application, Mechanical Engineering Research Studies: Engineering Dynamics Series, Research Studies Press Ltd, Hertfordshire, England, UK, 2nd edition, 2000. 


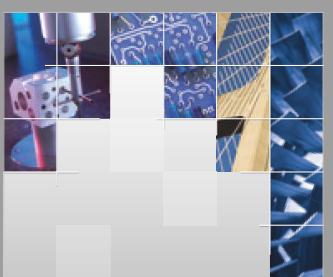

\section{Enfincering}
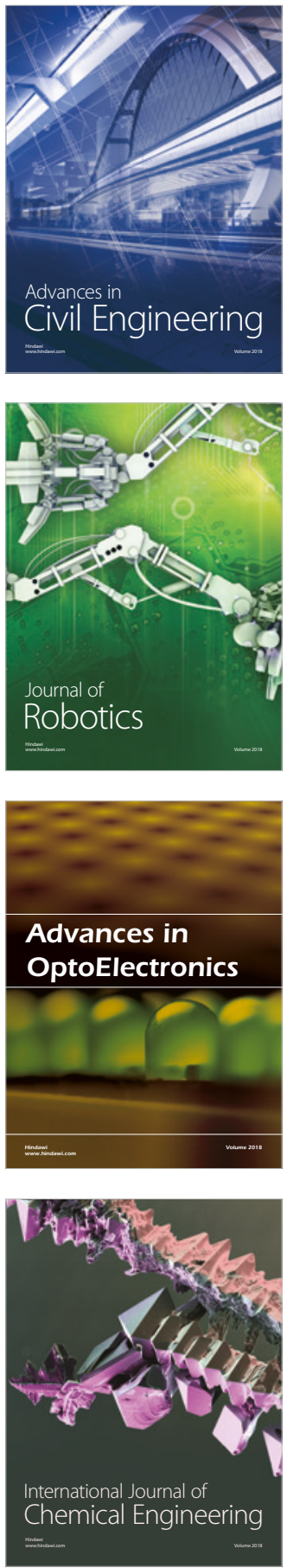

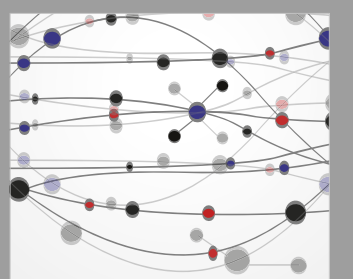

\section{Rotating \\ Machinery}

The Scientific World Journal

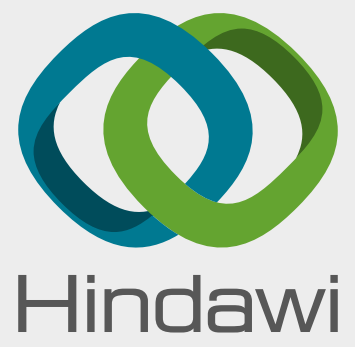

Submit your manuscripts at

www.hindawi.com
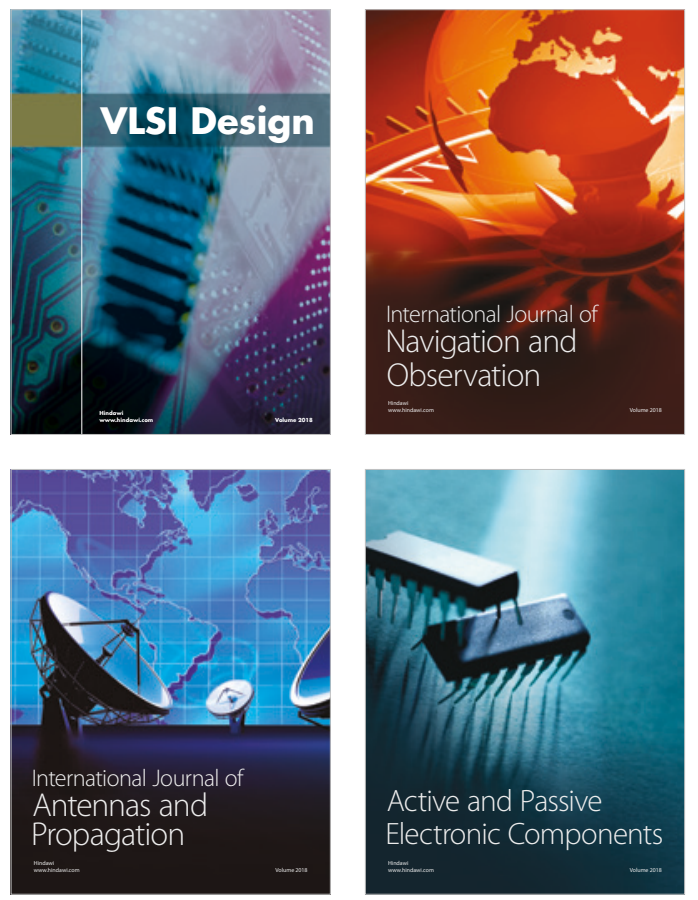
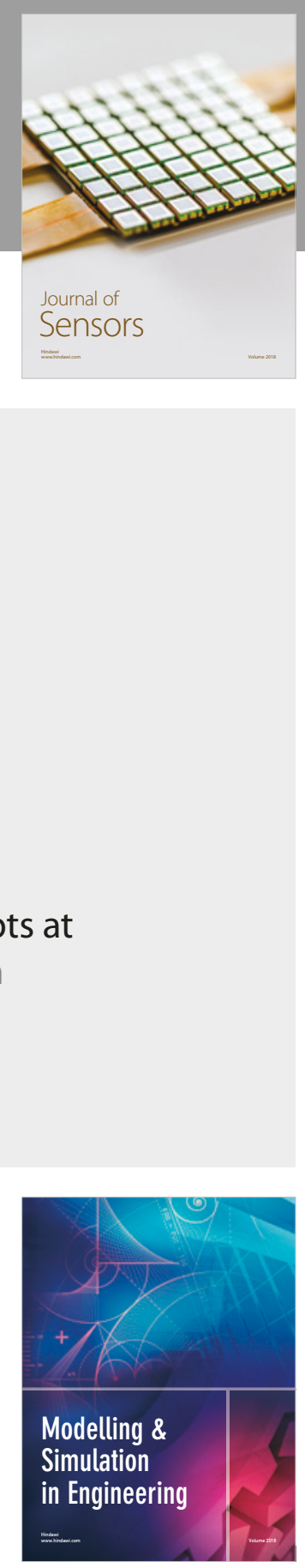

\section{Advances \\ Multimedia}
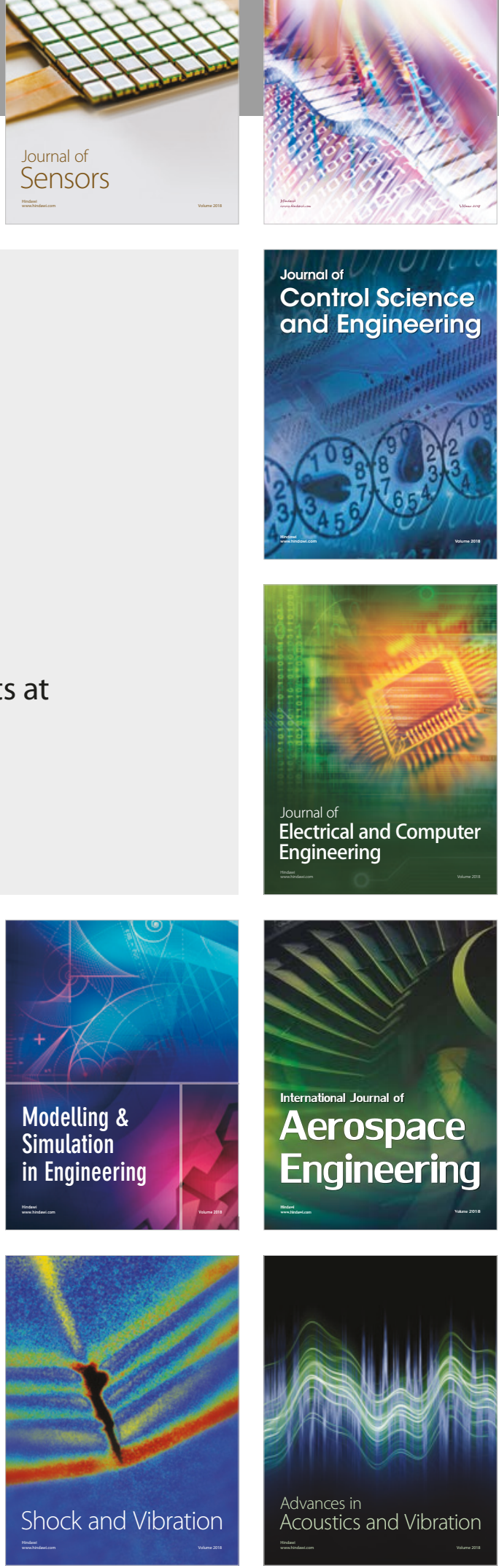